\title{
Improved Air Light Estimation Algorithm by using Fuzzy Filters and Dark Channel with Large Haze Gradients
}

\author{
Anil Rai \\ Department of Computer Science \& Engineering, \\ DAVIET Jalandhar, Punjab
}

\begin{abstract}
Fog phenomena bring about air flow gentle generating and also decline this awareness involving made from photograph caught in the camera. To increase awareness, air flow gentle evaluation is essential regarding photograph errors removal. As air flow gentle can be quite dazzling, this conventional methods immediately select dazzling $\mathrm{p}$ regarding air flow gentle estimation.In this paper improved/hybrid fuzzy filters based haze removal algorithm is proposed. The dark channel prior can automatically extract the global atmospheric light and roughly eliminate the atmospheric veil. To make dark channel prior more effective, the atmospheric veil has been refined by using hybrid fuzzy filters as well as it able to produce a haze free image in more optimistic manner. The use of improved/hybrid fuzzy filters has improved the coarse estimated atmospheric veil by reducing halo artifacts.
\end{abstract}

\section{Keywords}

Image defogging; Dark channel prior; Air light Estimation; Fuzzy filtering.

\section{INTRODUCTION}

Poor visibility turns into a problem for many out of doors eyesight applications. Negative climate brought on by atmospheric allergens, including haze, haze, etc., may considerably reduce the visibility and overlook the colors from the scene. The following as a result of the examples below 2 scattering techniques

(i) Light reflected from the surface of item can be attenuated caused by scattering by simply dust; and also

(ii) Some of light flux is definitely spread toward a camera.
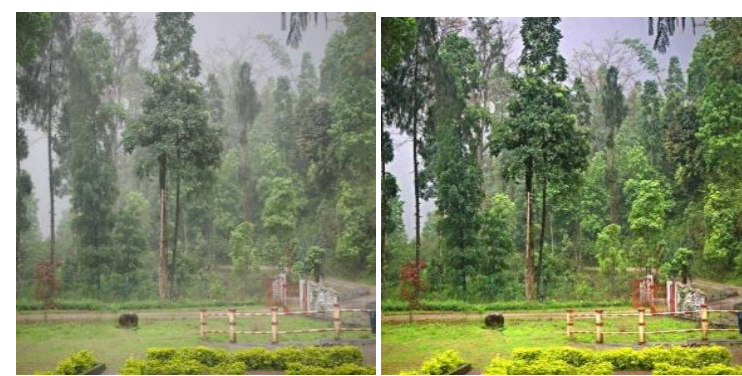

Figure 1: Results of image (a) with and (b) without fog/ Haze [12]

\subsection{Image Defogging Effect Assessment}

The CNC index, a powerful defogging review sign which often is beneficial in order to slowly move the parameter adjusting procedure [4]. With the source hazy image $\boldsymbol{x}$ and the unique equivalent fog free picture $\boldsymbol{y}$, the CNC index is received just after undertaking these kinds of steps:

\author{
Harpreet K. Bajaj \\ Assistant Professor \\ Department of Computer Science \& Engineering, \\ DAVIET Jalandhar, Punjab
}

1) Calculate the velocity $e$ of seen tips soon after as well as previous to haze elimination.

2) compute the picture colour naturalness index $(\mathrm{CNI})$ and colour colorfulness index (CCI) to measure the colour naturalness of fog free image $\mathbf{y}$, and

3) Merge several components $e$, CNI in addition to $\mathrm{CCI}$ in order to provide an overall defogging consequence.

$\mathrm{CNC}(\mathrm{x}, \mathrm{y})=h(e(\mathrm{x}, \mathrm{y}), \mathrm{CNI}(\mathrm{y}), \mathrm{CCI}(\mathrm{y})) \ldots \ldots(1)$

For the entire deviation trend of these three indexes, the statistical outcomes depict that the maximum value of CNI curve holds the normal outcome, but it is not inevitably the most effective fog removal impact. Nevertheless, the most effective outcome should have excellent naturalness (high $\mathrm{CNI}$ value). Once the picture is over-enhanced, the color is usually deformed, in addition to CNI fails swiftly. With regard to $e$ and $\mathrm{CCI}$, they have the most effective influence before getting to their particular highs. Once the picture is over-improved, the curves keep on climbing. Right after attaining the highs, these arcs begin to decrease. Hence, in event of the raising trend of $e$ and CCI (from their total capability result issues, hence, to their arc's peaks) often results in negating the falling-trend of CNI, as well as top of $\mathrm{CNC}$ arc may be close to the real most effective result level. At the same time, the actual value variant associated with $\mathrm{CNI}$ is usually compact, this is among one of $e$ and $\mathrm{CCI}$ is rather high. Hence, a result of $e$ and CCI around the CNC catalog ought to be lessened. This CNC index among graphic $\mathrm{x}$ and $\mathbf{y}$, i.e. the function $h$ in (1) may be considered as:

$\mathrm{CNC}(\mathbf{x}, \mathbf{y})=e(\mathbf{x}, \mathbf{y}) 1 / 5 \cdot \mathrm{CNI}(\mathbf{y})+\mathrm{CCI}(\mathbf{y}) 1 / 5 \cdot \mathrm{CNI}(\mathbf{y})$

As described above, a superb outcome can be tagged by the important valuation on CNC. Hence, the best outcome of the two main factors of defogging algorithm is found once the $\mathrm{CNC}$ index defines the greatest value.

\subsection{Visibility Restoration}

Restoring the particular scene albedo is definitely an inversion practice of your enhancement model of some sort of foggy and also obscure image. The consist of process may be decomposed in about three methods: calculate of your skylight, inference of your atmospheric veil $\mathrm{V}(\mathrm{x})$ on the scene image $\mathrm{I}(\mathrm{x})$ resolution of the scene albedo $\mathrm{p}(\mathrm{x})$ through inverting the following scattering model.

\subsection{Air Light Estimation}

The skylight A new is actually approximated on the pixel with many noteworthy power in the greater part of the past single picture strategies. The aggravating impacts of a white item prompt erroneous bay window estimation. In [8], the measure of the main channel indicates the bigger size to sift through a 
new white article having smaller sized measurements, nevertheless it will likewise wrongly get rid of a compact sky region. Since the truth in the skylight some sort of has an essential part inside the renewal practice, we all provide right here your better approach to locate the sun region. A minimum filter can be 1st conducted for the picture of the particular minimal aspect of $\mathrm{I}(\mathrm{x})$ as a way to remove insignificant sound and smaller light things, as well as output of the particular filter for just a pixel by can be denoted by simply (x). Subsequently, we all embrace the particular canny rider to be able to find perimeters of dull version to get exploding image. For each borders pixel, we all add up the particular relation concerning the volume of borders pixels and the full amount of pixels in it is smaller area to get a percentage place. The pixels that satisfy both $I_{\min }(\mathrm{x})>T_{\mathrm{v}}$ and $N_{\text {edge }}(x)<T_{\mathrm{p}}$ are selected to be candidates for the sky region. We fix the brightness threshold $T_{\mathrm{v}}$ to $95 \%$ of the maximum value of $I_{\min }(\mathrm{x})$ and the flatness threshold $T_{\mathrm{p}}$ to 0.001. Finally, we search for the first connected component from top to bottom and these pixels are determined as the sky region. The actual skylight can be believed while the absolute maximum importance of the related district inside the insight impression $\mathrm{I}(\mathrm{x})$

\subsection{Visibility Restoration Technique}

Regarding eliminating haze, haze with the graphic various tactics are usually used. Regular tactics involving graphic recovery towards haze are usually:

\subsubsection{Dark Channel Prior}

Dark channel prior can be useful for your opinion regarding atmospheric gentle within the dehazed impression to discover the much more real result. This approach is mainly useful for non-sky areas; in one coloring funnel have got nominal intensity from couple of pixels. Time frame intensity at midnight funnel can be main 3 factors:

- Shadows (shadows of car, buildings etc)

- Dark items or surfaces (dark tree trunk, stone)

- Colorful items, surfaces

For the reason that out-of-doors illustrations or photos are generally stuffed with eye shadows the dark stations of illustrations or photos are going to be really dark.

Because of errors (air light), a foggy photo is usually happier in comparison with the photo without the need of fog. So that we know dimly lit route involving foggy photo are going to have increased high intensity inside area using increased fog. So, aesthetically a power of dimly lit route is usually an uncertain appraisal with the thickness involving fog. Inside dimly lit route before we apply before along with write-up processing actions pertaining to obtaining results. Inside write-up processing actions we apply soft matting as well as trilateral filter etc.

\subsubsection{CLAHE}

Contrast restricted versatile histogram equalization small type is usually CLAHE. Contrast Limited Flexible Histogram Equalization (CLAHE) is usually used in advancement involving low distinction images. This procedure doesn't have almost any believed temperature details for any handling involving fogged image. To begin with, the style seized by means of you in foggy problem is usually converted via RGB (red, environment friendly along with blue) coloring living space is usually changed into HSV (hue, saturation along with value) coloring space. The pictures are generally converted due to the fact the human being sense colors in the same way while HSV characterize colors.

\subsubsection{Bilateral Filtering}

Bilateral filtration smoothes illustrations or photos looked after saves tips, having nonlinear combination of neighborhood impression values. Bilateral is definitely low iterative, community, and also simple. Grayish ranges or hues are usually merged by way of the bilateral separate out dependant on both its mathematical area along with their photometric related, and also likes close up values for you to distant values in the domain name and also range. Bilateral separate out sleek tips in the direction of piecewise consistent solutions. Bilateral separate out does not offer healthier sounds reduction.

\subsubsection{MIX - CLAHE}

The idea is a method to enhance under the sea photos employing a concoction Form a contrast Constrained Flexible Histogram Equalization. This augmentation technique successfully increases the presence involving under the sea photos and also provides the cheapest MSE and also the best PSNR values. Thus, it offers revealed the fact that mixCLAHE based method appealing intended for classifying coral reefs especially when aesthetic hints will be visible.

\subsubsection{Trilateral Filtering}

This specific filtration smooth's illustrations or photos without having impacting perimeters, by way of the non-linear mix of neighborhood photo values. Around the heap filter switches every pixel by weighted averages of that neighbor's pixel. The load allotted to each neighbor pixel lessens having the range in the photo airline and also the range to the severeness axis. This filter allows us to obtain result quicker while rival other. When using trilateral narrow all of us employ pre-processing along with publish producing ways with regard to greater results. Histogram stretch is utilized while post-processing along with histogram equalization as a before processing. [4]

\subsubsection{Fuzzy Filtering}

The idea driving this filtering is usually to normal your pixel using additional pixel ideals by reviewing the neighborhood, however simultaneously to address critical image structures just like edges. The main priority from the recommended filtering is usually to distinguish between regional variations as a result of noises in addition to as a result of image structure. Your order to accomplish this, each pixel all of us derive a price in which communicates their education where the mixture within a particular path is usually small. This kind of a price is usually derived each path equivalent on the nearby pixels from the refined pixel by way of fluffy rule. The further engineering from the filtering is then in line with the watching with interest which a modest fluffy mixture almost certainly is usually the result of noises, whilst a sizable fluffy mixture almost certainly is usually the result of a footing while in the image. Therefore, each path i will use not one but two fluffy regulations in which carry this specific watching with interest note, understanding that decide this contribution from the nearby pixel values. The consequence of these regulations is usually defuzzified plus a "a static correction term" is usually purchased for any refined pixel importance.

\section{RELATED WORK}

F. C. Cheng et al [7] examined blunders phenomena result in fresh air light-weight get older in addition changed for the worse the field of perspective together with coupled with effect grabbed inside camera. To further improve area associated with perspective, fresh air light-weight quote is 
essential suitable for effect errors removal. Whenever fresh air light-weight is reasonably brilliant, a fliers as well as business card printing instantly settle on brilliant pixels suitable for fresh air lumination estimation. K.B. Gibson et al. [12] discussed the way in which lots of simple picture defogging methods accomplish working with a color ellipsoid framework. That formula makes use of enhanced Gaussian union layout to enable you to take into account numerous combinations offering pure instinct in many challenging paying attention a glass windows, like observations in degree discontinuities which was 1 prevalent overuse injury in simple picture defogging. Zhiyuan Xu et al. [1] have tried to think about that may, the best ways movie press collection improved for the more serious just by faults endure negative visibility. A new variance minimal flexible histogram equalization (CLAHE)-based procedure began to remove fog. CLAHE determines some sort of the greatest worth so that you can sneak peek this histogram as well as redistributes this linked pixels just as so that you can each dreary level.Jing Yu et al. [15] mentioned that the image resolution along with lousy weather conditions are normally severely improved for your even worse by scattering resulting from dangling allergens in the establishing such as haze, problems in addition to mist. Your fresh new speedy defogging method from a single graphic of the world with different speedy bilateral filtration technique ended up utilized. Shih-Chia Huang et al. [14] discussed about the development coming from the concept of vision associated with ocean haze pictures in the unmanned expanse ship picture procedure, this work introduced some kind of publication defogging process based on a marriage strategy. The ultimate defogging result's got utilizing a simple enough bright firmness process. Ma, Zhongli et al. [20] discussed just about any excellent pixel answer to the prices within the skies additionally non-sky sections, that lets you mitigate your own halo bedroom throughout the sharpened tips lowering shading physique distortions above region. Wang et al. [4] used the multi-scale stiffness thoughts games formula in order to approximation that atmospheric veil, so that you can shape an awful strengthen plus sort the form a contrast regarding information during different scales. An item can bring about pitiable outcome after does not recognize your regional maxima in addition to local minima accurately. Zhiyuan et al. [2] discussed photographs transformed for that more serious using haze be afflicted by inferior contrast. So that you can get haze have an effect on, almost any Variety the contrast Small Multipurpose Histogram Equalization (CLAHE)-based approach became presented. Huimin Lu et al.[3] That record covers a different approach to bolster beneath the water graphics merely by photo dehazing. Scattering as well as coloring remodel are usually a list of critical complications associated with distortion with regards to beneath the water imaging. Scattering is definitely the end result of big shut down dust, such as turbid waters comprising abundant particles. This system resembles better than the state-of-theart approaches merely by if it turns out inside a lot of up-todate photo assessment systems. Zhiyuan et al. [5] Photographs deteriorated by means of errors are afflicted by undesirable contrast. So they can improve the difference, the errors deteriorated perception difference progression procedure based upon Bilinear Interpolation Powerful Histogram Equalization is in fact proposed. Sooner or later, HE and also Bilinear Interpolation usually are correspondingly executed on the image. Fresh effects demonstrate that the proposed procedure delivers a lot better procedure rather than range of standard algorithms. Shuai et al.[6] The particular is usually in order to appraisal a regular objective from the while using the media channels stations variety procedure prior to this candle lit station, to ensure media channels stations objective far better as well as complement any wiener variety closer. Your criteria however but not only compensates with regard to a deficiency associated with candle lit station previous criteria, but in addition increases this work associated with candle lit station previous criteria as well as lessens a functional time period around the photograph algorithm.

\section{METHODOLOGY}

1. In the initial phase, various underwater, remote sensing and road side images will be taken for experimental purpose from various datasets available on internet . and the format of the images will be jpg, png etc.

2.In this phase some well known existing algorithms will be designed and implemented using a suitable tool like MATLAB, JAVA etc

3. In this phase in order to improve the coarse estimated atmospheric veil by using different improved/hybrid filters will be implemented in order to remove the hallo artifacts and to preserve significant detail of restored images with large haze gradients

4.In this phase, the proposed technique will be tested and verified for the secondary data set of road side, underwater and remotely sensed images.

5. Finally, comparative analysis will be developed for the vision research community.

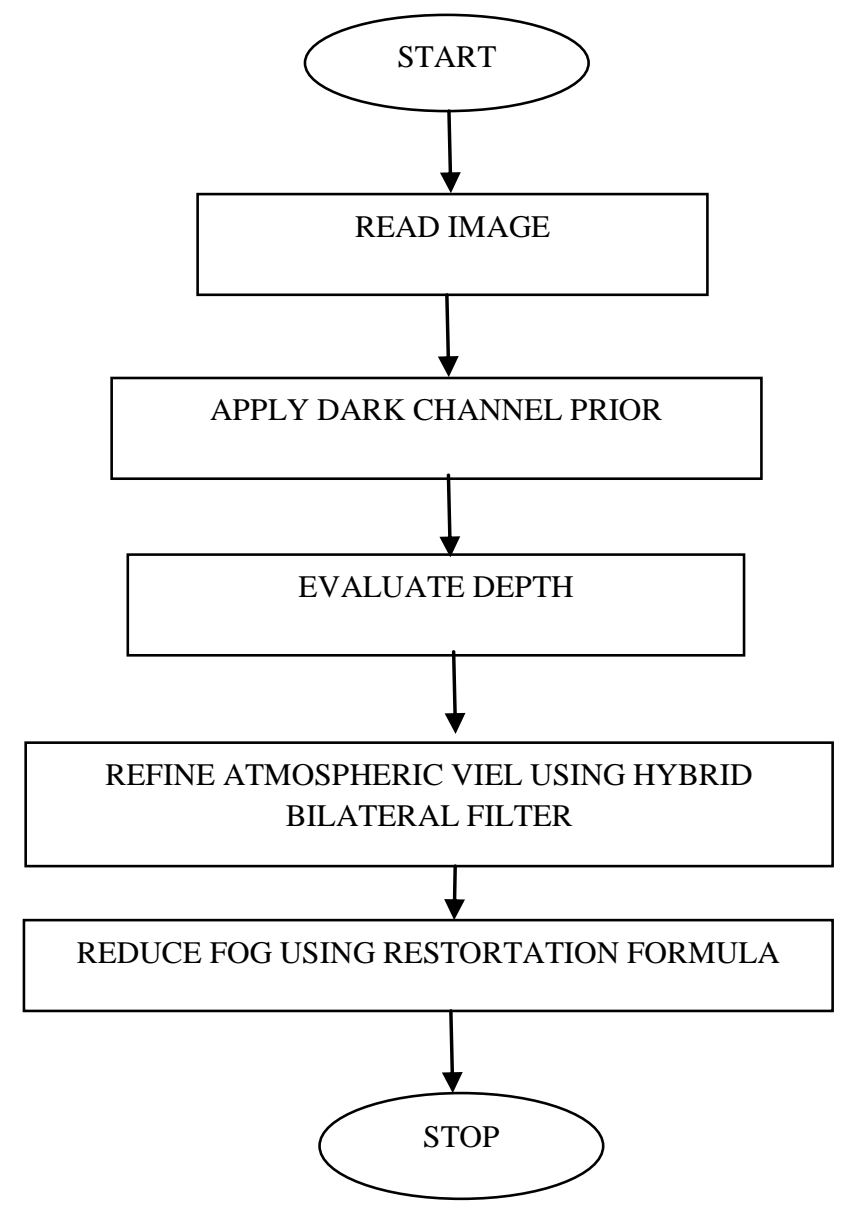

Fig 2. Flowchart of the proposed technique 


\section{RESULTS}

For experimentation and implementation the proposed technique is evaluated using MATLAB tool u2013a. The evaluation of proposed technique is done on the basis of following parameters i.e. Entropy, PSNR, MSE, based on different images.

1.

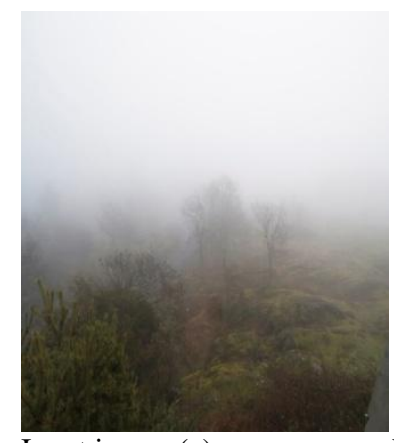

Input image (a)

Existing output image (b)

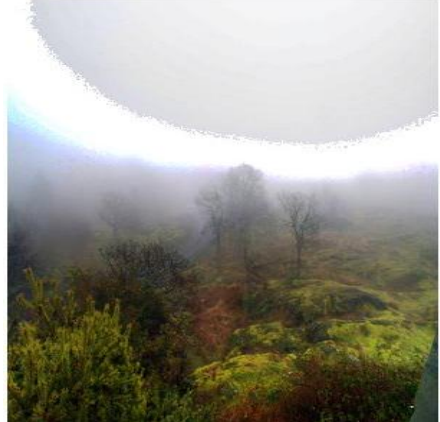

Proposed output image(c)

2

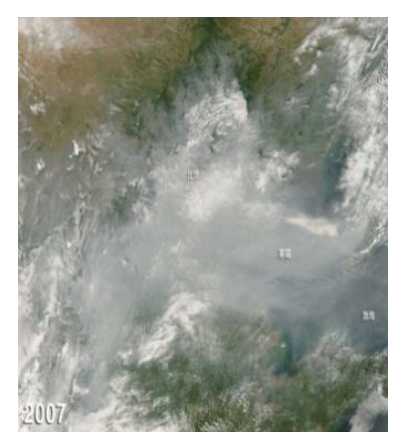

Input image (a)

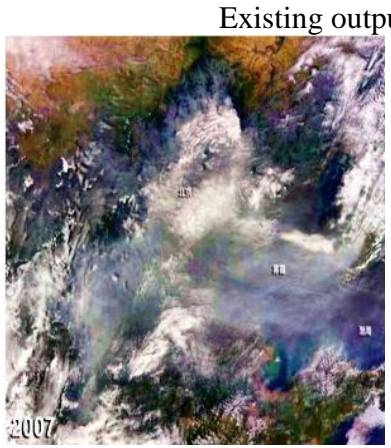

Proposed output image(c)

These foggy images have been taken from the google website the images are Landsat foggy image, modis foggy image and quick bird foggy image etc.
4.1. MSE (Mean Squared Error) -Mean square error will be to compute one indication through subtracting quality indication through the reference, after which it computing the standard power in the blunder signal. It can be explained as:

$M S E=\frac{1}{M N} \sum_{i=1}^{M} \sum_{j=1}^{N}\left(f(i, j)-f^{\prime}(i, j)\right)^{2}$

Where $\mathrm{j}(\mathrm{s})$ is the global normalized histogram of the processed image, $i$ is the brightness level index, pi is the probability of occurrence of level $i$ in the enhanced image [1].

\section{Table1. Mean Square Error}

\begin{tabular}{|c|c|c|}
\hline Images & $\begin{array}{c}\text { Mean Square } \\
\text { Error (existing) }\end{array}$ & $\begin{array}{c}\text { Mean Square } \\
\text { Error (proposed) }\end{array}$ \\
\hline Img1 & 0.0314 & 0.0069 \\
\hline Img2 & 0.0356 & 0.0198 \\
\hline Img3 & 0.0555 & 0.0119 \\
\hline Img4 & 0.0353 & 0.0091 \\
\hline Img5 & 0.0570 & 0.0211 \\
\hline Img6 & 0.0287 & 0.0072 \\
\hline Img7 & 0.0569 & 0.0185 \\
\hline Img8 & 0.0302 & 0.0205 \\
\hline Img9 & 0.0500 & 0.0200 \\
\hline Img10 & 0.0300 & 0.0244 \\
\hline
\end{tabular}

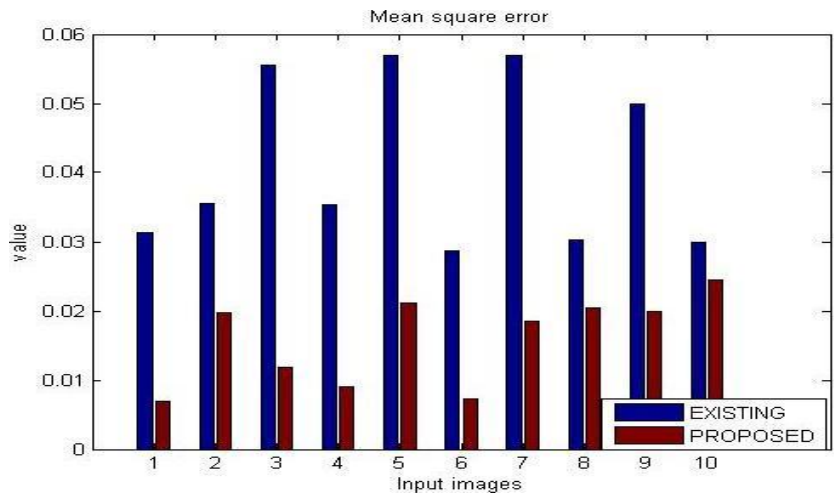

Fig3. Mean Square Error

\subsection{RMSE (Root Mean Square Error) -}

Root-mean-square error can be a measure on the differences between valuations forecast by means of one or maybe estimator as well as valuations basically observed. It can be explained as:

$$
R M S E=\sqrt{\frac{1}{M N} \sum_{i=1}^{M} \sum_{j=1}^{N}\left(f(i, j)-f^{\prime}(i, j)\right)^{2}}
$$

Table2. Root Mean Square Error

\begin{tabular}{|l|l|l|}
\hline Images & $\begin{array}{l}\text { Root Mean Square } \\
\text { Error (existing) }\end{array}$ & $\begin{array}{l}\text { Root Mean Square } \\
\text { Error (proposed) }\end{array}$ \\
\hline Img1 & 0.1773 & 0.0833 \\
\hline Img2 & 0.1887 & 0.1406 \\
\hline Img3 & 0.2355 & 0.1089 \\
\hline
\end{tabular}




\begin{tabular}{|l|l|l|}
\hline Img4 & 0.1879 & 0.0953 \\
\hline Img5 & 0.2388 & 0.1454 \\
\hline Img6 & 0.1694 & 0.0849 \\
\hline Img7 & 0.2384 & 0.1359 \\
\hline Img8 & 0.1737 & 0.1430 \\
\hline Img9 & 0.3000 & 0.1413 \\
\hline Img10 & 0.1733 & 0.1563 \\
\hline
\end{tabular}

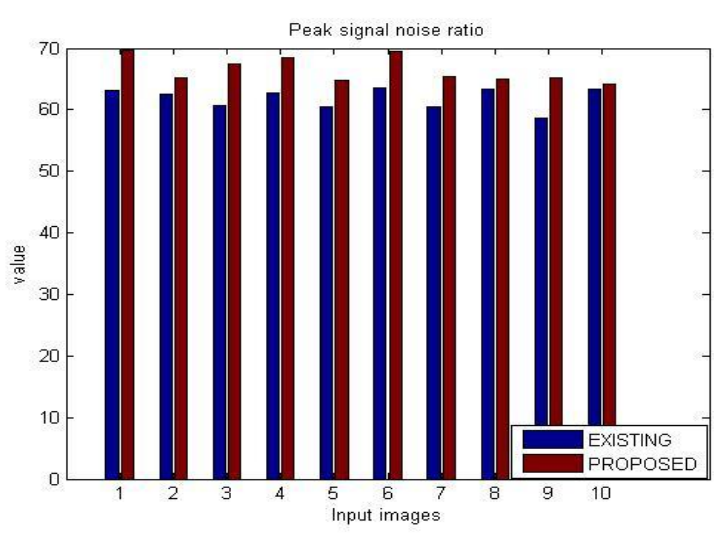

Fig4. Bit Error Rate

\subsection{Cross-Correlation Evaluation}

Cross-Correlation (CC) should to be close to1, therefore planned algorithm is showing better effects compared to the available techniques as CC is near to 1 in every case.

Table 4.Cross Correlation Evaluation

\begin{tabular}{|c|c|c|}
\hline Image name & $\begin{array}{c}\text { Existing } \\
\text { algorithm }\end{array}$ & $\begin{array}{c}\text { Proposed } \\
\text { algorithm }\end{array}$ \\
\hline image 1 & 0.6076 & 0.9842 \\
\hline image 2 & 1.1131 & 0.9753 \\
\hline image3 & 0.5935 & 0.9666 \\
\hline image4 & 0.5662 & 1.2876 \\
\hline image5 & 1.2214 & 0.8041 \\
\hline image6 & 0.9077 & 0.8874 \\
\hline image7 & 0.6215 & 0.9523 \\
\hline image8 & 0.6978 & 0.9726 \\
\hline image9 & 1.0178 & 0.8557 \\
\hline image10 & 1.1631 & 1.1040 \\
\hline
\end{tabular}

Table3. Bit Error Rate

\begin{tabular}{|c|c|c|}
\hline Images & Bit Error Rate (existing) & $\begin{array}{c}\text { Bit Error Rate } \\
\text { (proposed) }\end{array}$ \\
\hline Img1 & 0.0158 & 0.0143 \\
\hline Img2 & 0.0160 & 0.0153 \\
\hline Img3 & 0.0165 & 0.0148 \\
\hline Img4 & 0.0160 & 0.0146 \\
\hline Img5 & 0.0165 & 0.0154 \\
\hline Img6 & 0.0157 & 0.0144 \\
\hline Img7 & 0.0165 & 0.0153 \\
\hline Img8 & 0.0158 & 0.0154 \\
\hline Img9 & 0.0171 & 0.0154 \\
\hline Img10 & 0.0158 & 0.0156 \\
\hline
\end{tabular}

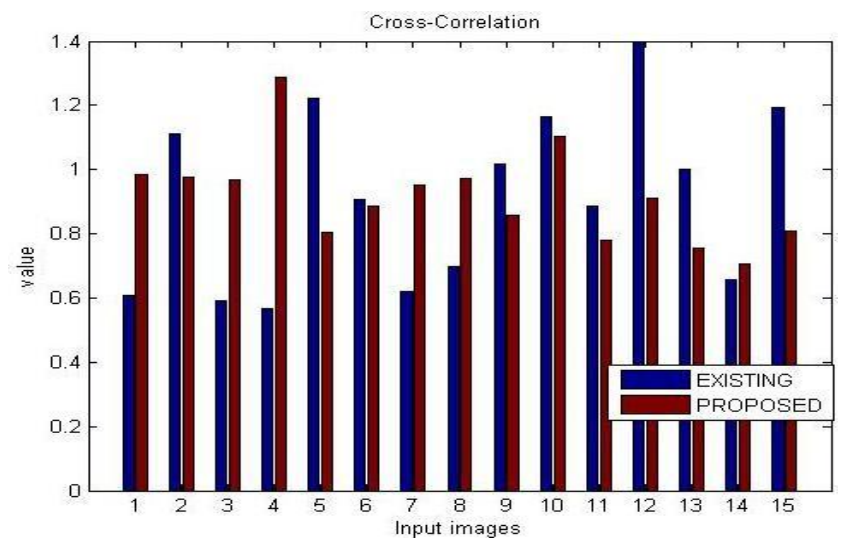

Fig 5. CC for the existing and the proposed method

Figure 7.3 is showing the quantized analysis of the CrossCorrelation of different images using existing method (Pink Color), proposed method (blue Color). It is very clear from the plot that value of $\mathrm{CC}$ is close tol in every case with the use of proposed method over other methods. This represents improvement in the objective quality of the image. 


\subsection{Median Angular Error}

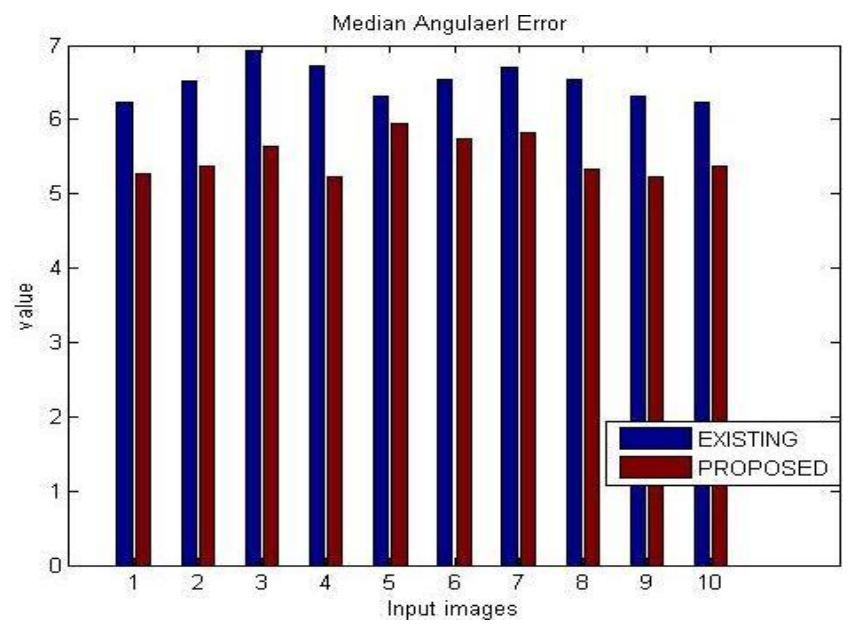

Fig6. Median Angular Error

Table5. Root Mean Square Error

\begin{tabular}{|c|c|c|}
\hline Image name & $\begin{array}{c}\text { Existing } \\
\text { algorithm }\end{array}$ & $\begin{array}{c}\text { Proposed } \\
\text { algorithm }\end{array}$ \\
\hline image 1 & 6.2374 & 5.2731 \\
\hline image 2 & 6.5273 & 5.3732 \\
\hline image3 & 6.9372 & 5.6348 \\
\hline image4 & 6.7323 & 5.2342 \\
\hline image5 & 6.3231 & 5.9372 \\
\hline image6 & 6.5432 & 5.7321 \\
\hline image7 & 6.6933 & 5.8333 \\
\hline image8 & 6.5381 & 5.3217 \\
\hline image9 & 6.3212 & 5.2372 \\
\hline image10 & 6.2312 & 5.3723 \\
\hline
\end{tabular}

\section{CONCLUSION}

This paper has proposed the improved air light estimation algorithm by using fuzzy filters and dark channel prior to preserve significant detail of restored images with large haze gradients. It has been designed and implemented in Matlab tool 2013.The comparison of various parameters like Peak signal to noise ratio, Median angular error, Mean square error, Root mean square error, Cross correlation has been taken which has been improved the coarse estimated atmospheric veil by reducing halo artifacts as well as it is able to produce the haze free image in more optimistic manner.

\section{REFERENCES}

[1] Atta, Randa, and Rabab Farouk Abdel-Kader. "Brightness preserving based on singular value decomposition for image contrast enhancement." OptikInternational Journal for Light and Electron Optics 126, no. 7 (2015): 799-803.

[2] Bhandari, A. K., Anil Kumar, G. K. Singh, and Vivek Soni. "Dark satellite image enhancement using knee transfer function and gamma correction based on DWTSVD." Multidimensional Systems and Signal Processing (2015): 1-24.
[3] Pathak SS, Dahiwale P, Padole G. A combined effect of local and global method for contrast image enhancement. In Engineering and Technology (ICETECH), 2015 IEEE International Conference on 2015 Mar 20 (pp. 1-5). IEEE.

[4] Ghosh, Soham, Sourya Roy, Utkarsh Kumar, and Arijit Mallick. "Gray Level Image Enhancement Using Cuckoo Search Algorithm." In Advances in Signal Processing and Intelligent Recognition Systems, pp. 275-286. Springer International Publishing, 2014.

[5] Ji X, Cheng J, Bai J, Zhang T, Wang M. Real-time enhancement of the image clarity for traffic video monitoring systems in haze. In Image and Signal Processing (CISP), 2014 7th International Congress on 2014 Oct 14 (pp. 11-15). IEEE

[6] Agarwal TK, Tiwari M, Lamba SS. Modified histogram based contrast enhancement using homomorphic filtering for medical images. In Advance Computing Conference (IACC), 2014 IEEE International 2014 Feb 21 (pp. 964968). IEEE

[7] Negi SS, Bhandari YS. A hybrid approach to Image Enhancement using Contrast Stretching on Image Sharpening and the analysis of various cases arising using histogram. In Recent Advances and Innovations

[8] Bouaziz A, Draa A, Chikhi S. A Cuckoo search algorithm for fingerprint image contrast enhancement. In Complex Systems (WCCS), 2014 Second World Conference on 2014 Nov 10 (pp. 678-685). IEEE.

[9] Mathew, Ammu Anna, and S. Kamatchi. "Brightness and Resolution Enhancement of Satellite Images using SVD and DWT." International Journal of Engineering Trends and Technology 4, no. 4 (2013): 712-718.

[10] Gupta, Nidhi, and Rajib Jha. "Enhancement of High Dynamic Range Dark Images Using Internal Noise in DWT Domain." In Intelligent Interactive Technologies and Multimedia, pp. 66-74. Springer Berlin Heidelberg, 2013.

[11] Huang, Shih-Chia and Chien-Hui Yeh. "Image contrast enhancement for preserving mean brightness without losing image features." Engineering Applications of Artificial Intelligence 26, no. 5 (2013): 148

[12] Xie, Zhihua. "Single sample face recognition based on dct and local Gabor binary pattern histogram." In Intelligent Computing Theories, pp. 435-442. Springer Berlin Heidelberg, 2013.

[13] Lee, Edward, Sungho Kim, Wei Kang, Daeban Seo, and Jamie Paik. "Contrast enhancement using dominant brightness level analysis and adaptive intensity transformation for remote sensing images." Geoscience and Remote Sensing Letters, IEEE 10, no. 1 (2013): 6266.

[14] Wen, Haocheng, Yonghong Tian, Tiejun Huang, and Wen Gao "Single underwater image enhancement with a new optical model." In Circuits and Systems (ISCAS), 2013 IEEE International Symposium on, pp. 753-756. IEEE, 2013.

[15] Nercessian SC, Panetta K, Agaian SS. Non-linear direct multi-scale image enhancement based on the luminance and contrast masking characteristics of the human visual 
system. Image Processing, IEEE Transactions on. 2013 Sep; 22(9):3549-61.

[16] Huynh-The T, Le-Tien T. Brightness preserving weighted dynamic range histogram equalization for image contrast enhancement. In Advanced Technologies for Communications (ATC), 2013 International Conference on 2013 Oct 16 (pp. 386-391). IEEE.

[17] Kotkar VA, Gharde SS. Image contrast enhancement by preserving brightness using global and local features. In Computational Intelligence and Information Technology, 2013. CIIT 2013. Third International Conference on 2013 Oct 18 (pp. 262-271). IET.

[18] Bhandari AK, Gadde M, Kumar A, Singh GK. Comparative analysis of different wavelet filters for low contrast and brightness enhancement of multispectral remote sensing images. In Machine Vision and Image Processing (MVIP), 2012 International Conference on 2012 Dec 14 (pp. 81-86). IEEE.

[19] Gupta, Kanika, and Akshu Gupta. "Image enhancement using ant colony optimization." IOSR J. VLSI Signal Process 1 (2012): 38.

[20] Khan, Nafis Uddin, K. V. Arya, and Manisha Pattanaik. "A New Adaptive Thresholding in SVD for Efficient Image De-noising." In Proceedings of the International Conference on Soft Computing for Problem Solving (SocProS 2011) December 20-22, 2011, pp. 659-670. Springer India, 2012. 